\title{
Measurement of the branching ratio of $\pi^{0}$ Dalitz decay using $K_{L} \rightarrow \pi^{0} \pi^{0} \pi^{0}$ decays
}

E. Abouzaid, ${ }^{4}$ M. Arenton, ${ }^{11}$ A. R. Barker, ${ }^{5,}$ L. Bellantoni, ${ }^{7}$ E. Blucher, ${ }^{4}$ G. J. Bock, ${ }^{7}$ E. Cheu, ${ }^{1}$ R. Coleman, ${ }^{7}$ M. D. Corcoran, ${ }^{9, *}$ B. Cox, ${ }^{11}$ A. R. Erwin, ${ }^{12, *}$ C. O. Escobar, ${ }^{3}$ A. Glazov, ${ }^{4,}$ A. Golossanov, ${ }^{11}$ R. A. Gomes, ${ }^{3}$ P. Gouffon, ${ }^{10}$ Y. B. Hsiung, ${ }^{7}$ D. A. Jensen, ${ }^{7}$ R. Kessler, ${ }^{4}$ K. Kotera, ${ }^{8}$ A. Ledovskoy, ${ }^{11}$ P. L. McBride, ${ }^{7}$ E. Monnier, ${ }^{4,}$ H. Nguyen, ${ }^{7}$ R. Niclasen, ${ }^{5}$ D. G. Phillips II ${ }^{11}$ E. J. Ramberg, ${ }^{7}$ R. E. Ray ${ }^{7}$ M. Ronquest,${ }^{11}$ E. Santos, ${ }^{10}$ W. Slater, ${ }^{2}$ D. Smith, ${ }^{11}$ N. Solomey, ${ }^{4}$ E. C. Swallow, ${ }^{4,6, *}$ P. A. Toale, ${ }^{5}$ R. Tschirhart, ${ }^{7}$ Y. W. Wah, ${ }^{4}$ J. Wang, ${ }^{1}$ H. B. White, ${ }^{7}$ J. Whitmore, ${ }^{7}$ M. J. Wilking, ${ }^{5}$ B. Winstein, ${ }^{4,}{ }^{*}$ R. Winston, ${ }^{4}$ E. T. Worcester, ${ }^{4,8}$ T. Yamanaka, ${ }^{8}$ E. D. Zimmerman, ${ }^{5}$ and R. F. Zukanovich ${ }^{10}$

${ }^{1}$ University of Arizona, Tucson, Arizona 85721, USA

${ }^{2}$ University of California at Los Angeles, Los Angeles, California 90095, USA

${ }^{3}$ Universidade Estadual de Campinas, Campinas 13083-970, Brazil

${ }^{4}$ The Enrico Fermi Institute, The University of Chicago, Chicago, Illinois 60637, USA

${ }^{5}$ University of Colorado, Boulder, Colorado 80309, USA

${ }^{6}$ Elmhurst College, Elmhurst, Illinois 60126, USA

${ }^{7}$ Fermi National Accelerator Laboratory, Batavia, Illinois 60510, USA

${ }^{8}$ Osaka University, Toyonaka, Osaka 560-0043, Japan

${ }^{9}$ Rice University, Houston, Texas 77005, USA

${ }^{10}$ Universidade de São Paulo, São Paulo 05315-970, Brazil

${ }^{11}$ The Department of Physics and Institute of Nuclear and Particle Physics, University of Virginia, Charlottesville, Virginia 22901, USA

${ }^{12}$ University of Wisconsin, Madison, Wisconsin 53706, USA

(Received 6 February 2019; published 5 August 2019)

\begin{abstract}
We present a measurement of $B\left(\pi^{0} \rightarrow e^{+} e^{-} \gamma\right) / B\left(\pi^{0} \rightarrow \gamma \gamma\right)$, the Dalitz branching ratio, using data taken in 1999 by the E832 KTeV experiment at Fermi National Accelerator Laboratory. We use neutral pions from fully reconstructed $K_{L}$ decays in flight; the measurement is based on $\sim 60$ thousand $K_{L} \rightarrow \pi^{0} \pi^{0} \pi^{0} \rightarrow$ $\gamma \gamma \gamma \gamma e^{+} e^{-} \gamma$ decays. We normalize to $K_{L} \rightarrow \pi^{0} \pi^{0} \pi^{0} \rightarrow 6 \gamma$ decays. We find $B\left(\pi^{0} \rightarrow e^{+} e^{-} \gamma\right) / B\left(\pi^{0} \rightarrow \gamma \gamma\right)$ $\left(m_{e^{+} e^{-}}>15 \mathrm{MeV} / c^{2}\right)=[3.920 \pm 0.016($ stat $) \pm 0.036(\mathrm{syst})] \times 10^{-3}$. Using the Mikaelian and Smith prediction for the $e^{+} e^{-}$mass spectrum, we correct the result to the full $e^{+} e^{-}$mass range. The corrected result is $B\left(\pi^{0} \rightarrow e^{+} e^{-} \gamma\right) / B\left(\pi^{0} \rightarrow \gamma \gamma\right)=[1.1559 \pm 0.0047$ (stat) \pm 0.0106 (syst) $] \%$. This result is consistent with previous measurements, and the uncertainty is a factor of 3 smaller than any previous measurement.
\end{abstract}

DOI: $10.1103 /$ PhysRevD.100.032003

\section{INTRODUCTION}

The neutral pion decays electromagnetically to two photons with a branching ratio of $\sim 99 \%$. The next most common decay mode, $\pi^{0} \rightarrow e^{+} e^{-} \gamma$, was first suggested by Richard Dalitz in 1951. He calculated the leading order (QED) decay rate relative to $\pi^{0} \rightarrow \gamma \gamma$ to be

\footnotetext{
${ }^{*}$ Deceased.

†Permanent address: DESY, Hamburg, Germany.

${ }^{\ddagger}$ Permanent address: C.P.P. Marseille/C.N.R.S., Paris, France. USA.

${ }^{\S}$ Permanent address: Brookhaven National Lab, Upton, NY,

Published by the American Physical Society under the terms of the Creative Commons Attribution 4.0 International license. Further distribution of this work must maintain attribution to the author(s) and the published article's title, journal citation, and DOI. Funded by SCOAP.
}

$B\left(\pi^{0} \rightarrow e^{+} e^{-} \gamma\right) / B\left(\pi^{0} \rightarrow \gamma \gamma\right)=1.185 \%$ [1]. Radiative corrections to the Dalitz decay rate have since been calculated to order $\alpha^{2}$ and predict $B\left(\pi^{0} \rightarrow e^{+} e^{-} \gamma\right) / B\left(\pi^{0} \rightarrow \gamma \gamma\right)=$ $1.196 \%$ [2-7]. A recent calculation using alternative methods reports $B\left(\pi^{0} \rightarrow e^{+} e^{-} \gamma\right) / B\left(\pi^{0} \rightarrow \gamma \gamma\right)=(1.1978 \pm$ $0.0006) \%$ [8]. The Dalitz decay branching fraction has previously been measured to be $B\left(\pi^{0} \rightarrow e^{+} e^{-} \gamma\right) / B\left(\pi^{0} \rightarrow\right.$ $\gamma \gamma)=(1.188 \pm 0.035) \%$ [9]. The Dalitz decay is used as a normalization mode for a number of rare kaon and pion decays, and the $\sim 3 \%$ uncertainty in the Dalitz branching ratio measurement is a limiting factor for many of these measurements.

This paper reports a new measurement of the Dalitz decay rate using $K_{L} \rightarrow \pi^{0} \pi^{0} \pi^{0}$ decays in which one of the three pions decays to $e^{+} e^{-} \gamma\left(K_{L} \rightarrow 3 \pi_{D}^{0}\right) . K_{L} \rightarrow$ $\pi^{0} \pi^{0} \pi^{0}$ decays in which all three pions decay to two photons are used for normalization. The measurement is 
based on $63,693 K_{L} \rightarrow 3 \pi_{D}^{0}$ decays collected from June to September 1999 by the KTeV experiment at the Fermi National Accelerator Laboratory (FNAL). In Sec. II, we describe the $\mathrm{KTeV}$ beam, experimental apparatus, event reconstruction, and data analysis techniques. In Sec. III, we describe the Dalitz branching ratio analysis, including corrections to the branching ratio and systematic uncertainties. Section IV contains the branching ratio result and crosschecks of that result. Section V provides a comparison to other results and the new world average.

\section{THE KTEV EXPERIMENT}

In the $\mathrm{KTeV}$ experiment, two neutral kaon beams were produced by a proton beam incident on a target. The $800-\mathrm{GeV} / \mathrm{c}$ proton beam, provided by the Fermilab Tevatron, had a 53-MHz radio frequency structure so that the protons arrived in $\sim 1$-ns wide "buckets" at 19-ns intervals. The proton extraction cycle was 40-s extractions every $80 \mathrm{~s}$. About half of the data collected in 1999 was at an average intensity of $1.6 \times 10^{11}$ protons/s with the other half collected at a lower intensity of about $1 \times 10^{11}$ protons/s as a systematic cross-check. The primary purpose of the $\mathrm{KTeV}$ experiment was the measurement of $\operatorname{Re}\left(\epsilon^{\prime} / \epsilon\right)$ [10]. For this reason, a "regenerator" was placed in one of the beams to produce a source of $K_{S}$ decays; this beam is called the regenerator beam and the other beam is called the vacuum beam. For the Dalitz branching ratio analysis, we use only $K_{L}$ decays from the vacuum beam. A charged spectrometer was used to measure the momenta and trajectories of charged particles, while the cesium iodide (CsI) calorimeter was used to measure the positions and energies of photons and electrons. A veto system was used to reject background and a three-level trigger was used to select events. A detailed Monte Carlo simulation was used to predict the acceptance difference between the signal and normalization decay modes, and to study background. The following sections give a brief description of the $\mathrm{KTeV}$ detector and reconstruction techniques; these are described in more detail in [10-12].

\section{A. The KTeV detector}

The $\mathrm{KTeV}$ kaon beams were produced by an $800 \mathrm{GeV} / c$ proton beam, provided by the FNAL Tevatron, incident on a beryllium oxide $(\mathrm{BeO})$ target that was about one proton interaction length long. In the $\mathrm{KTeV}$ coordinate system, the positive $z$-axis points downstream with its origin at the target. The two beams were shaped and the nonkaon content was reduced by a beamline of magnets, absorbers, and collimators. There was an evacuated decay region surrounded by lead-scintillator photon veto detectors from 90 to $160 \mathrm{~m}$ downstream of the target. After the vacuum decay region, there were the charged spectrometer, trigger

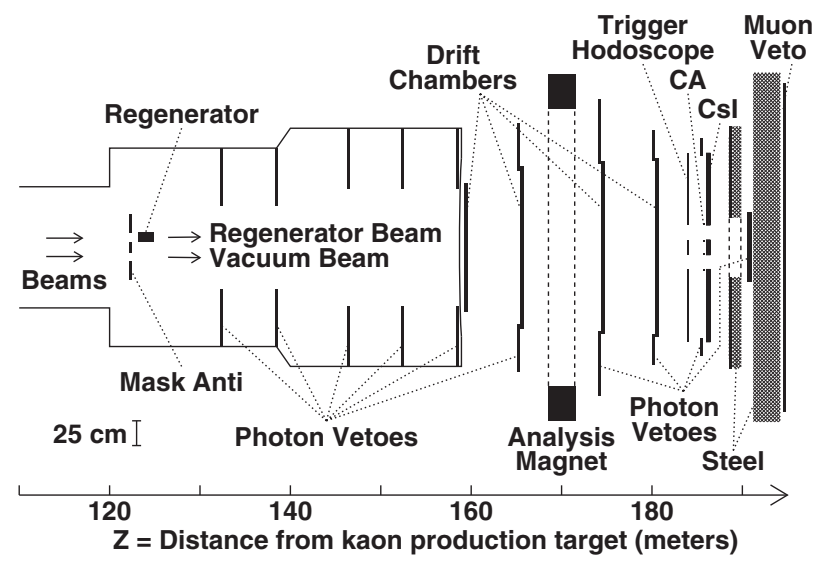

FIG. 1. Schematic of the KTeV detector. Note that the vertical and horizontal scales are different.

hodoscope, CsI calorimeter, and muon veto systems. Figure 1 is a schematic of the detector.

The charged spectrometer was composed of four drift chambers at $z=159 \mathrm{~m}, z=166 \mathrm{~m}, z=175 \mathrm{~m}$, and $z=$ $181 \mathrm{~m}$, and a dipole analyzing magnet at $z=170 \mathrm{~m}$. Each drift chamber consisted of four planes of sense wires, two were horizontal and two were vertical. Each sense wire was surrounded by six field-shaping wires, resulting in a hexagonal cell geometry in each plane. The electron drift velocity was $\sim 50 \mu \mathrm{m} / \mathrm{ns}$ in the equal-parts argon-ethane gas mixture inside the drift chambers; this corresponds to a maximum drift time across each cell of $150 \mathrm{~ns}$. The two planes of sense wires in each view were offset from each other by half a cell to resolve the left-right ambiguity. The magnet produced a field which was uniform to better than $1 \%$ and provided a $0.41 \mathrm{GeV} / c$ momentum kick in the horizontal plane. The known kaon mass was used to set the momentum scale with $10^{-4}$ precision.

The CsI calorimeter was composed of 3100 pure cesium iodide crystals that were each viewed by a photomultiplier tube. The CsI crystals in the inner region of the calorimeter were $2.5 \times 2.5 \mathrm{~cm}^{2}$ in the transverse plane, and the crystals in the outer region were $5.0 \times 5.0 \mathrm{~cm}^{2}$. The crystals were all $50 \mathrm{~cm}$ (27 radiation lengths) long; therefore, most of the energy from photons and electrons hitting the CsI calorimeter was measured by the detector. Two square, carbonfiber beam holes allowed the beams to pass through the calorimeter. Momentum analyzed electrons and positrons from $K_{L} \rightarrow \pi^{ \pm} e^{\mp} \nu$ decays were used to calibrate the CsI energy scale to $0.02 \%$.

A three-level trigger was used to select events during data collection. Level 1 used fast signals from the detector, Level 2 was based on processing from custom electronics, and Level 3 was a software filter. One of the Level 2 processors was the hardware cluster counter (HCC), which counted isolated clusters of energy in the CsI calorimeter. The $K_{L} \rightarrow 3 \pi_{D}^{0}$ decays for this analysis were selected by a trigger that required seven or more HCC clusters, while the $K_{L} \rightarrow \pi^{0} \pi^{0} \pi^{0}$ events for the normalization mode were 
selected by a different trigger requiring six or more $\mathrm{HCC}$ clusters.

\section{B. Event reconstruction}

Track reconstruction is performed by combining "hits" into "tracks." A hit is defined as an analog signal in a drift chamber sense wire that is above TDC threshold and is intime with the trigger signal. The hits in the two $x$ or $y$ planes of a drift chamber are called a hit-pair. For each hit-pair, the sum of drift distances (SOD) should be equal to the cell size, assuming a track that is perpendicular to the drift chamber and perfect resolution. Hit-pairs are required to have a SOD within $1 \mathrm{~mm}$ of the $6.35-\mathrm{mm}$ nominal cell width after correcting for the incident angle of the particle.

Track segments are constructed separately from hit-pairs in the two drift chambers upstream of the magnet and the two drift chambers downstream of the magnet; these segments are then extrapolated to the center of the magnet. We require that the extrapolated track segments match to within $6 \mathrm{~mm}$ at the magnet mid-plane. Each particle momentum is determined from the track bend-angle in the magnet and a map of the magnetic field. If two $x$ and $y$ tracks are found, we extrapolate both sets of tracks upstream to define an $x-z$ and a $y-z$ vertex. The difference between these two projections, $\Delta z_{\mathrm{vtx}}$, is used to define a vertex $\chi^{2}$,

$$
\chi_{\mathrm{vtx}}^{2} \equiv\left(\Delta z_{\mathrm{vtx}} / \sigma_{\Delta z}\right)^{2}
$$

where $\sigma_{\Delta z}$ is the resolution of $\Delta z_{\mathrm{vtx}}$. A track is required to have $\chi_{\mathrm{vtx}}^{2}$ less than 100. To determine the full particle trajectory, the $x$ and $y$ tracks are matched to each other based on their projections to the CsI calorimeter. Each extrapolated track position must match the position of a CsI calorimeter cluster to within $7 \mathrm{~cm}$.

The energies of photons and electrons are determined by measuring the energy deposited in the CsI calorimeter by electromagnetic showers. We define a "cluster" as a $7 \times 7$ array of small crystals or a $3 \times 3$ array of large crystals. Each cluster is centered on a "seed" crystal which contains the maximum energy deposit among crystals in the cluster. The energies in all the crystals in the cluster are summed; this sum is then corrected to account for partial clusters, energy leakage outside the cluster, energy shared between clusters, and nonuniform detector response.

To reconstruct the decay vertex from clusters in the CsI calorimeter, we group pairs of photons and determine which pairing produces the most consistent values for the decay vertex. For each photon pairing, we calculate $d_{12}$, the distance in $z$ between the $\pi^{0}$ decay vertex and $z_{\mathrm{CSI}}$, the mean shower depth in the CsI crystals. Using the pion mass as a constraint, in the small angle approximation, we find the distance from the CsI calorimeter to the vertex for each pair of photons to be

$$
d_{12} \approx \frac{\sqrt{E_{1} E_{2}}}{m_{\pi^{0}}} r_{12},
$$

where $r_{12}$ is the transverse distance between the two photons at the CsI calorimeter. For each pairing, we compare the calculated distances for each candidate pion. The consistency of the reconstructed distances is quantified using the pairing chi-squared variable $\left(\chi_{\pi^{0}}^{2}\right)$ which is based on the reconstructed vertex positions and resolutions. We choose the pairing that gives the minimum value of $\chi_{\pi^{0}}^{2}$ and require that this value be less than 75 .

Each pion in the signal and normalization modes decays either to two photons or to $e^{+} e^{-} \gamma$. We use $\chi_{\pi^{0}}^{2}$ to determine which particles come from the same pion. In $K_{L} \rightarrow 3 \pi_{D}^{0}$ events, there are two tracks and seven clusters; two of the clusters are paired to tracks leaving five clusters which must be separated into two pairs of photons from the two $\pi^{0} \rightarrow \gamma \gamma$ decays and one photon from the $\pi^{0} \rightarrow e^{+} e^{-} \gamma$ decay. In $K_{L} \rightarrow$ $\pi^{0} \pi^{0} \pi^{0}$ events, there are six clusters which must be paired.

The $x$ and $y$ positions of the kaon decay vertex are calculated using the reconstructed positions and energies of the clusters in the CsI calorimeter. In the case of tracks from the $\pi^{0} \rightarrow e^{+} e^{-} \gamma$ decay, the cluster positions are adjusted based on the upstream track segment, since the cluster's actual position in the calorimeter is the result of the track bending in the magnet. The CsI cluster energies and the decay vertex position are used to calculate the invariant mass for the $3 \pi_{D}^{0}$ and $3 \pi^{0}$ decays.

\section{Monte Carlo simulation}

We use the $\mathrm{KTeV}$ Monte Carlo (MC) simulation to determine the acceptance difference between the signal and normalization modes and to study background. The Monte Carlo simulates $K^{0} / \overline{K^{0}}$ generation at the $\mathrm{BeO}$ target following the parametrization in [13], propagates the coherent $K^{0} / \overline{K^{0}}$ state through the absorbers and collimators along the beamline to the decay point, simulates the decay, traces the decay products through the detector, and simulates the detector response including the digitization of the detector signals and the trigger selection. The parameters of the detector geometry are based both on data and survey measurements. Many aspects of the tracing and detector response are based on samples of detector responses, called "libraries," that are generated with GEANT3 [14] simulations.

The effects of accidental activity are included in the simulation by overlaying data events from an accidental trigger onto the simulated events. The accidental events used in the simulation are collected concurrently with the signal and normalization data, so that variations in accidental activity with changes in beam intensity are simulated by the MC. After veto requirements are applied, the average accidental energy contained in each CsI calorimeter cluster is a few $\mathrm{MeV}$, and there are about 20 extra in-time drift chamber hits in each event [11]. 
Simulation of inefficiencies and systematic effects in the drift chambers is crucial for the Dalitz branching ratio measurement. Individual wire inefficiencies, high-SOD hit-pairs from delayed hits, accidental hits that obscure hit signals, and low-SOD pairs from delta rays are all simulated by the MC.

We simulate both real and virtual radiative corrections to the $\pi^{0} \rightarrow e^{+} e^{-} \gamma$ decay. QED processes up to order $\alpha^{2}$ are included in the simulation; both real corrections, in which a photon radiates from one of the electrons, and virtual corrections, in which one-loop terms interfere with the treelevel diagram, are included. Real radiative $\pi^{0} \rightarrow e^{+} e^{-} \gamma \gamma$ events are generated for $m_{\gamma \gamma}$ greater than $1 \mathrm{MeV}$; below this threshold the real radiative process is indistinguishable in the $\mathrm{KTeV}$ detector from the tree-level process. A real radiative photon above the $1 \mathrm{MeV}$ threshold is generated in about $16 \%$ of the events. The virtual corrections are based on [4], which provides numerical results for radiative corrections over the full kinematic range of the $e^{+} e^{-}$mass and the energy partition between the electron and positron. A two-dimensional look-up table binned in the Kroll-Wada $x$ and $y$ variables, which are functions of the $e^{+} e^{-}$mass and the electron-positron energy partition, respectively, is used to select the appropriate correction factor.

The Monte Carlo event format is identical to data, and the events are reconstructed and analyzed in the same manner as data. More details of the simulation are available in [12].

\section{BRANCHING RATIO ANALYSIS}

To measure the Dalitz branching ratio, we collect $K_{L} \rightarrow$ $\pi^{0} \pi^{0} \pi^{0}$ events in which one of the three pions undergoes Dalitz decay $\left(K_{L} \rightarrow 3 \pi_{D}^{0}\right)$. The normalization mode is $K_{L} \rightarrow \pi^{0} \pi^{0} \pi^{0}$ in which each of the three pions decays to two photons. The detector acceptance for $K_{L} \rightarrow 3 \pi_{D}^{0}$ and $K_{L} \rightarrow \pi^{0} \pi^{0} \pi^{0}$ decays is quite different, so we use the Monte Carlo simulation to determine the $z$-dependent acceptance for each mode. We correct this acceptance for data-MC differences related to tracking inefficiencies and relative trigger differences between the two modes, and we assign systematic uncertainties associated with these corrections. We study additional sources of systematic uncertainty on the branching ratio measurement, such as our simulation of radiative corrections and interactions in the detector material. In Sec. III A, we describe the selection of signal and normalization events. In Sec. III B, we describe our determination of the relative acceptance of the two modes, the corrections we make to that acceptance, and the associated systematic uncertainties. Section III C describes the remaining sources of systematic uncertainty on the branching ratio measurement. A summary of the systematic uncertainties is given in Sec. III D.

\section{A. Event selection}

The $K_{L} \rightarrow 3 \pi_{D}^{0}$ signal and $K_{L} \rightarrow \pi^{0} \pi^{0} \pi^{0}$ normalization mode events are selected by separate triggers that require seven or more clusters and six or more clusters, respectively, in the CsI calorimeter. To ensure consistency between the signal and normalization modes, any runs or spills that do not contain both types of triggers are excluded.

We apply a number of selection criteria to the $K_{L} \rightarrow 3 \pi_{D}^{0}$ and $K_{L} \rightarrow \pi^{0} \pi^{0} \pi^{0}$ events. These cuts are chosen to avoid event topologies that have poor reconstruction efficiencies or that are difficult to simulate, to reduce backgrounds, and to define the acceptance. We keep the selection criteria for signal and normalization events as similar as possible so that any associated systematic uncertainties cancel in the ratio of the two modes. For those requirements associated with the tracks in the $\pi^{0} \rightarrow e^{+} e^{-} \gamma$ decay, we vary each requirement in both data and $\mathrm{MC}$ events to verify that the data are well-simulated by the $\mathrm{MC}$ in the region of that cut.

We eliminate signal events with more than two tracks and both signal and normalization events with extra clusters in the CsI calorimeter. In both modes, we require that the reconstructed invariant mass be within $7 \mathrm{MeV} / c^{2}$ of the known kaon mass, that the reconstructed $z$ vertex position be between 123 and $158 \mathrm{~m}$, and that the reconstructed kaon energy be between 40 and $160 \mathrm{GeV}$.

To avoid events in which the CsI calorimeter clusters are difficult to reconstruct and simulate, we place a number of requirements on the CsI clusters for both data and Monte Carlo. The minimum cluster energy in an event must be greater than $3 \mathrm{GeV}$, and the minimum distance between clusters must be greater than $7.5 \mathrm{~cm}$. The "ring number" variable [10], which describes the distance between the center of energy and the nearest beam hole, must be less than $110 \mathrm{~cm}^{2}$. Events in which the kaon decay occurred within one of the neutral beams should have a ring number less than $86.5 \mathrm{~cm}^{2}$ [10]. The $\chi_{\gamma}^{2}$ variable, which describes how close a cluster's transverse energy distribution is to the distribution expected for a photon shower [15], must be less than 100 . We remove events in which one of the clusters has its seed crystal in the innermost or outermost ring of crystals in the CsI calorimeter. These criteria are identical for both the signal and normalization modes.

A number of additional requirements are placed on the $K_{L} \rightarrow 3 \pi_{D}^{0}$ signal mode to select events in which the tracks are well-reconstructed and simulated. We require that the reconstructed $e^{+} e^{-} \gamma$ invariant mass be within $20 \mathrm{MeV} / c^{2}$ of the known $\pi^{0}$ mass. The resolution of the $e^{+} e^{-} \gamma$ invariant mass is $\sim 1.5 \mathrm{MeV} / c^{2}$. The minimum track momentum must be greater than $4.0 \mathrm{GeV} / c$, and the ratio of measured energy in the CsI cluster associated with the track to the measured track momentum, $\mathrm{E} / p$, must be greater than 0.9 . 
We remove events in which a track passes too close to the edge of a detector. These fiducial cuts reduce the sensitivity of the measurement to our knowledge of the physical size and location of these detectors. The tracks must be more than $2-3 \mathrm{~mm}$ away from inner edges of the veto detectors and trigger hodoscope that surround the neutral beams and at least $2.9 \mathrm{~cm}$ away from the outer edge of the CsI calorimeter.

We remove events in which one of the electrons emits a bremsstrahlung photon as it bends in the magnet. The bremsstrahlung photon is typically emitted parallel to the direction of the electron prior to bending in the magnet. To identify these events, we project the upstream segment of each track to the CsI calorimeter and identify the closest photon cluster. The distance between the track projection and the position of the nearest cluster is called the "brem- $\gamma$ distance"; we require this quantity to be greater than $1 \mathrm{~cm}$.

A critical requirement on $K_{L} \rightarrow 3 \pi_{D}^{0}$ events is that the $e^{+} e^{-}$tracks be separated by more than three drift chamber cells in the two upstream drift chambers. This corresponds to a distance requirement of more than $\sim 2 \mathrm{~cm}$. The MC simulation of tracks in the same or neighboring cells, complicated by other effects such as accidental hits, delta rays, and high-SOD pairs, is difficult; this cell separation requirement is necessary to ensure that the tracking efficiency is well-modeled.

We also require that the reconstructed $e^{+} e^{-}$mass be greater than $15 \mathrm{MeV} / c^{2}$; this requirement is related to the cell separation requirement because small values of $e^{+} e^{-}$ mass correspond to close tracks. Since the cell separation requirement removes most events with a reconstructed $e^{+} e^{-}$mass less than $10 \mathrm{MeV} / c^{2}$, the analysis requirement on $e^{+} e^{-}$mass cleanly defines the kinematic region of our measurement by excluding the region where the acceptance is very small. Additionally, the low $e^{+} e^{-}$mass region is more sensitive to real and virtual radiative corrections than the region above $15 \mathrm{MeV} / c^{2}$. Removing the low mass region reduces the sensitivity of this measurement to theoretical predictions and allows the measurement to be updated in the future when new calculations of radiative corrections at low $e^{+} e^{-}$mass are available.

\section{B. Acceptance and acceptance corrections}

The detector acceptance for $K_{L} \rightarrow 3 \pi_{D}^{0}$ and $K_{L} \rightarrow$ $\pi^{0} \pi^{0} \pi^{0}$ decays is the ratio of simulated events passing all reconstruction criteria to the total number of events simulated. Monte Carlo events are generated in a larger kinematic range than will be accepted by the analysis, so that the possibility of event migration across selection criteria boundaries is treated correctly. The MC simulation includes kaon decays in the range $110 \mathrm{~m}<z<161 \mathrm{~m}$ and $35 \mathrm{GeV}<E<165 \mathrm{GeV}$. Dalitz decays are simulated for all possible $e^{+} e^{-}$masses.

We simulate $\sim 200$ million $K_{L} \rightarrow 3 \pi_{D}^{0}$ decays and $\sim 300$ million $K_{L} \rightarrow \pi^{0} \pi^{0} \pi^{0}$ decays. Out of these, the fraction of Dalitz events accepted by the analysis is $(1.1714 \pm 0.0023) \times 10^{-3}$. The fraction of events accepted for $K_{L} \rightarrow \pi^{0} \pi^{0} \pi^{0}$ decays is $(3.7853 \pm 0.0010) \times 10^{-2}$. The quoted uncertainties are from MC statistics only. There are two differences between the data and MC that have a significant effect on the acceptance calculation: the simulation of tracking efficiencies in the signal mode, and the simulation of the relative trigger efficiencies between the signal and normalization modes. We correct the acceptance determined from Monte Carlo for these known differences between data and the Monte Carlo simulation. The following sections describe how we determine the appropriate acceptance correction for each of these discrepancies.

\section{Acceptance correction for tracking efficiencies}

In Dalitz decay, the angle between the electron and positron tends to be small, so the track separation in the two upstream drift chambers is small. It is difficult to simulate tracking inefficiency for close tracks, so we must correct for data-MC differences in tracking inefficiency.

We use an independent sample of $K_{L} \rightarrow \pi^{+} \pi^{-} \pi^{0}$ decays to measure the tracking efficiency for two charged particles in the $\mathrm{KTeV}$ detector because these events can be reconstructed without full tracking. We reconstruct the decay vertex position of the $\pi^{0} \rightarrow \gamma \gamma$ decay using the positions and energies of clusters in the CsI calorimeter. The two charged pions are associated with hadronic showers in the CsI calorimeter; these are differentiated from the photon clusters using the $\chi_{\gamma}^{2}$ variable. We then use this sample to measure the single-track and two-track inefficiencies in data and Monte Carlo as described below.

To measure the single-track inefficiency, we require one of the hadronic clusters in the CsI calorimeter to match a fully reconstructed track. There are two possible kinematic solutions for the second track; the position of the second hadronic cluster resolves the ambiguity. The single-track inefficiency, $\eta_{1}$, is half the ratio of events with a missing track to the total number of events; the factor of 2 is included since either of the two tracks could be lost.

To measure the probability of failing to reconstruct both tracks, $\eta_{0}$, we select events in which there are two track segments in either the upstream or downstream pair of drift chambers, but no complete tracks are reconstructed. The ratio of these events to the total is the two-track inefficiency.

These tracking efficiency measurements are performed in both data and Monte Carlo samples at two different beam intensities, where the average intensities in the two samples differ by about a factor of 2 . The measured inefficiencies are summarized in Table I. The data-MC differences for medium and high intensity data are averaged to apply a correction of $-0.68 \%$ to the acceptance calculation.

Most of the measured tracking inefficiency is due to accidental activity. To demonstrate this, we perform the tracking efficiency measurements on Monte Carlo events that do not include accidental overlays and find that the 
TABLE I. Tracking inefficiencies in $K_{L} \rightarrow \pi^{+} \pi^{-} \pi^{0}$ data and Monte Carlo, for two different beam intensities. The correction applied to the acceptance is the difference between the total data inefficiency and the total MC inefficiency.

\begin{tabular}{lcc}
\hline \hline & \multicolumn{2}{c}{ Tracking inefficiency } \\
\cline { 2 - 3 } & Medium intensity & High intensity \\
\hline Data & $3.48 \%$ & \\
$2 \eta_{1}$ & $0.19 \%$ & $4.90 \%$ \\
$\eta_{0}$ & $3.67 \%$ & $0.21 \%$ \\
Total & & $5.11 \%$ \\
Monte Carlo & $2.97 \%$ & \\
$2 \eta_{1}$ & $0.05 \%$ & $4.31 \%$ \\
$\eta_{0}$ Total & $3.02 \%$ & $0.09 \%$ \\
Correction & $0.65 \%$ & $4.40 \%$ \\
\hline \hline
\end{tabular}

measured inefficiencies in this sample are much smaller than in the nominal Monte Carlo. We find that $~ 90 \%$ of the measured tracking inefficiency results from accidentals. The impact of accidental activity is well-modeled in the MC and the observed data-MC discrepancy is largely independent of intensity, as seen in Table I. We conclude that much of the $-0.68 \%$ correction to the efficiency cannot be attributed to accidental activity. Since this discrepancy is unexplained, we assign a systematic uncertainty on the Dalitz branching ratio measurement that is equal to the full size of the correction to the efficiency.

\section{Acceptance correction for relative trigger efficiencies}

The $K_{L} \rightarrow 3 \pi_{D}^{0}$ signal and $K_{L} \rightarrow \pi^{0} \pi^{0} \pi^{0}$ normalization events are selected with different triggers. To measure the relative inefficiencies between the two triggers, we use a rescaled sample of events from the normalization mode trigger that do not have any Level 3 requirements applied. We apply the $K_{L} \rightarrow 3 \pi_{D}^{0}$ reconstruction algorithm and selection criteria to this sample and search for events that would be included in the Dalitz analysis but are not included in the sample selected by the Dalitz trigger. All requirements are the same as in the primary analysis except that the cell separation cut is removed to increase statistics. In this sample of 716 events, we find one event that passes all other Dalitz selection criteria but is not included in the Dalitz sample. The same study is performed on Monte Carlo events with no measurable relative trigger inefficiency found. This dataMonte Carlo difference in trigger inefficiency of $0.14 \%$ is applied as a correction to the acceptance. We assign a systematic uncertainty on the Dalitz branching ratio measurement that is equal to the size of the correction.

We also measure the absolute inefficiency of the trigger used to select $K_{L} \rightarrow \pi^{0} \pi^{0} \pi^{0}$ decays for the normalization sample. We study data from a minimum bias trigger and

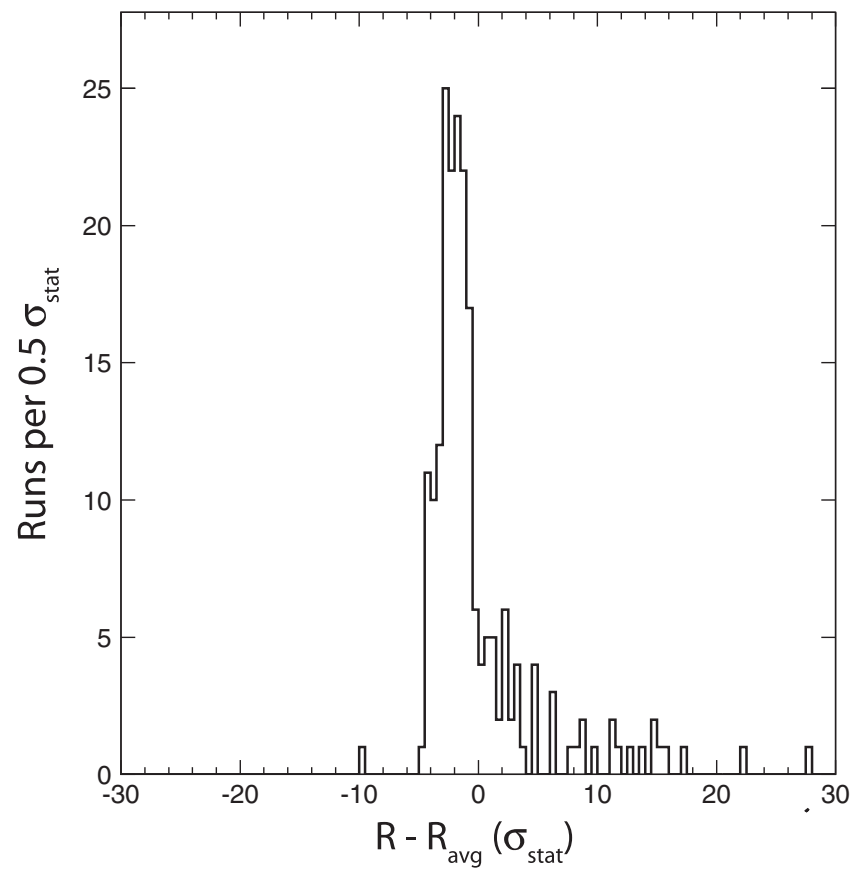

FIG. 2. Distribution of difference from the average value for ratio of number of events before to number of events after hardware prescale is applied, in units of statistical standard deviations.

search for events that would be accepted by the $K_{L} \rightarrow$ $\pi^{0} \pi^{0} \pi^{0}$ analysis but were not selected by the $K_{L} \rightarrow \pi^{0} \pi^{0} \pi^{0}$ trigger. We select a sample of $\sim 500,000 K_{L} \rightarrow \pi^{0} \pi^{0} \pi^{0}$ decays from the minimum bias trigger and measure the trigger inefficiency to be $(0.0042 \pm 0.0010) \%$. There is no trigger inefficiency simulated in the Monte Carlo, so the full inefficiency is a data-MC bias. We do not correct for this small inefficiency. We apply the standard $\mathrm{KTeV}$ procedure for setting systematic uncertainties that includes the statistical precision of the study [11]; we find a systematic uncertainty in the Dalitz branching ratio measurement of $0.0047 \%$.

A prescale of 2 at the hardware level and of $5 / 2$ at the software level is applied to the $K_{L} \rightarrow \pi^{0} \pi^{0} \pi^{0}$ trigger; there is no prescale applied to the $K_{L} \rightarrow 3 \pi_{D}^{0}$ sample. Any deviation of the prescale from the nominal values will produce a bias in the branching ratio measurement. The software prescale has no inaccuracy. We study the hardware prescale accuracy using scaler counts of the number of events before and after the hardware prescale was applied during data collection. For each individual run and for all runs combined, we calculate the ratio of events, $\mathrm{R}=$ $\mathrm{N}_{f} / \mathrm{N}_{i}$, after prescaling $\left(\mathrm{N}_{F}\right)$ to before prescaling $\left(\mathrm{N}_{i}\right)$, and the statistical uncertainty of each ratio. The average ratio is $R_{\mathrm{avg}}=0.500044 \pm 0.000003$. In Fig. 2, we plot the number of statistical sigmas from average for each run. We find a number of runs in which the measured prescale is significantly different from the nominal value; this indicates a small, intermittent defect in the prescale electronics. As $\sim 90 \%$ of runs have a discrepancy of less than $5 \sigma$, 
we assign an uncertainty of 5 times the statistical error on the total ratio. We therefore determine the prescale ratio to be $0.500044 \pm 0.000016$, which corresponds to systematic uncertainty in the branching ratio measurement of $<0.01 \%$.

\section{Other systematic uncertainties}

This section contains descriptions of how we assign the remaining systematic uncertainties for the measurement of $B\left(\pi^{0} \rightarrow e^{+} e^{-} \gamma\right) / B\left(\pi^{0} \rightarrow \gamma \gamma\right)$. All of the systematic uncertainties are summarized in Sec. III D.

\section{Radiative corrections}

As described in Sec. II C, the Monte Carlo simulation of the $\pi^{0} \rightarrow e^{+} e^{-} \gamma$ decay includes radiative corrections to second order in $\alpha_{\mathrm{EM}}$. The reconstructed $e^{+} e^{-} \gamma$ mass distribution is sensitive to the real corrections, while the reconstructed $e^{+} e^{-}$mass distribution is sensitive to the virtual corrections; data-MC comparisons for these quantities are shown in Figs. 3 and 4. Since the QED calculations that are used by the simulation are wellunderstood and data-MC comparisons indicate that the data are well-described by the simulation, we assign a systematic uncertainty on the Dalitz branching ratio due only to higher-order corrections that are not simulated. The acceptance change between Monte Carlo with no radiative corrections and the nominal $\mathrm{MC}$ with second order
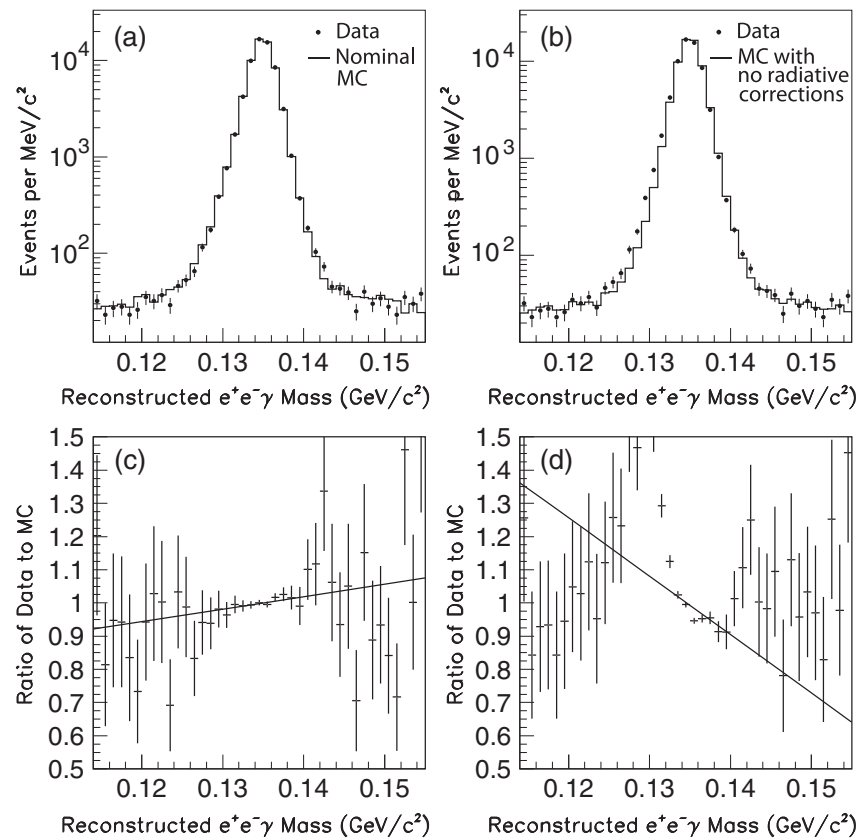

FIG. 3. Reconstructed $e^{+} e^{-} \gamma$ mass for data (dots) and Monte Carlo (histogram). (a) Data and nominal MC. (b) Data and $\mathrm{MC}$ with no radiative corrections. (c) Data/MC ratio for nominal MC. (d) Data/MC ratio for $\mathrm{MC}$ with no radiative corrections. All nominal selection criteria have been applied except for the $e^{+} e^{-} \gamma$ mass requirement.
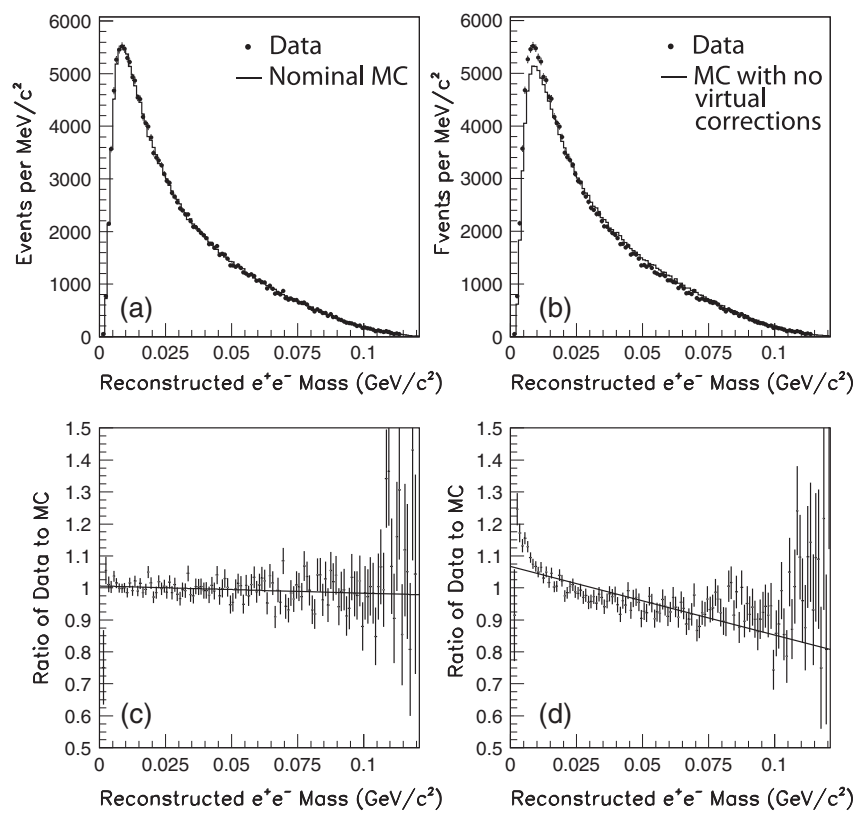

FIG. 4. Reconstructed $e^{+} e^{-}$mass for data (dots) and Monte Carlo (histogram). (a) Data and nominal MC. (b) Data and $\mathrm{MC}$ with no virtual radiative corrections. (c) Data/MC ratio for nominal MC. (d) Data/MC ratio for $\mathrm{MC}$ with no virtual radiative corrections. All nominal selection criteria have been applied except for the $e^{+} e^{-}$mass requirement and the cell separation requirement. These plots include events with minimum cell separation values of one or greater.

corrections is $-5.43 \%$. We assume that adding the next order of corrections would cause the same percentage change in acceptance; we therefore take $5.43 \%$ of $5.43 \%$, or $0.29 \%$, to be the systematic uncertainty in the branching ratio measurement due to higher-order radiative corrections.

\section{Detector material}

The Monte Carlo simulation includes bremsstrahlung radiation in the 0.018 radiation lengths of detector material located upstream of the final drift chamber. The simulation of bremsstrahlung in the nominal Monte Carlo changes the signal mode acceptance by $-4.66 \%$ relative to a MC with no bremsstrahlung simulation. The amount of detector material is known to about $10 \%$, so we assign a systematic uncertainty on the branching ratio measurement of $0.47 \%$.

\section{Accidental activity}

In addition to being the primary source of tracking inefficiency, accidental activity can affect the branching ratio measurement by adding extra tracks or CsI calorimeter clusters to an event. Accidentals affect the signal and normalization modes differently because tracks are present only in the signal mode, and because the normalization mode has one more photon in the final state than the signal mode. The presence of accidental overlays in the 

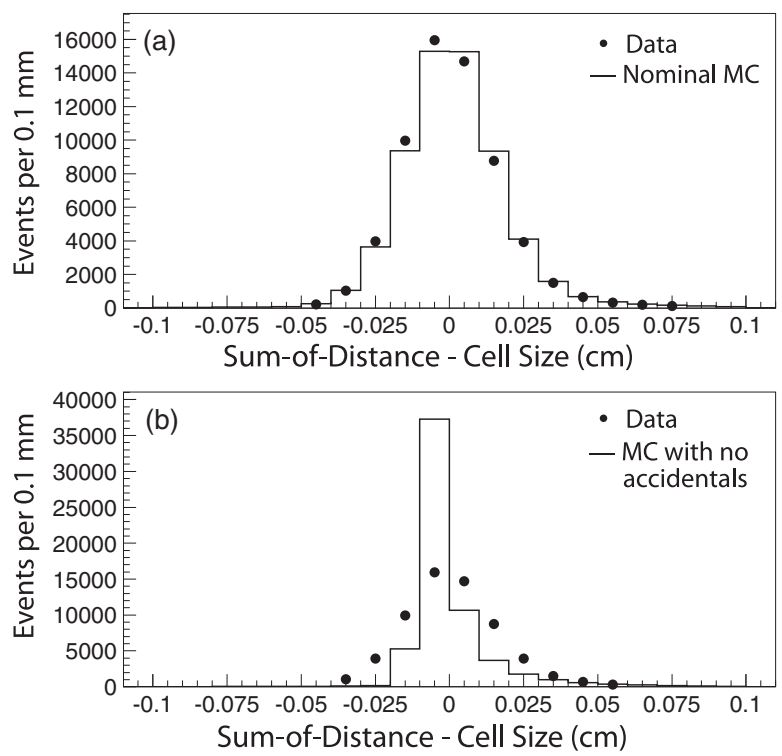

FIG. 5. Deviation of sum-of-distance (SOD) from nominal value of $6.35 \mathrm{~mm}$ for data (dots) and Monte Carlo (histogram). Note the different vertical scales for the two plots. (a) Data and nominal MC. (b) Data and MC with no simulation of accidental activity.

Monte Carlo simulation decreases the acceptance relative to a simulation with no accidentals by $37 \%$ for the signal mode and by $32 \%$ for the normalization mode. These changes largely cancel in the branching ratio measurement; the change in the branching ratio from the simulation of accidental activity is $3.96 \%$. We estimate the uncertainty in the branching ratio analysis from accidental activity by combining Monte Carlo samples as described in the following paragraphs.

To estimate the sensitivity of the branching ratio measurement to extra tracks from accidentals, we study events with low sum-of-distances (SOD) values because these events come almost entirely from accidentals. As shown in Fig. 5, the fraction of events with low SODs is wellmodeled by the Monte Carlo simulation; the "low-SOD fraction," defined as the fraction of events with SOD less than $-0.2 \mathrm{~mm}$, in the nominal MC is within 2.5 sigma of the fraction in data. To quantify the sensitivity of the measurement to our simulation of accidentals, we create Monte Carlo samples in which some fraction of events do not contain accidental overlays and find the level at which we can detect a data-MC discrepancy. We find that a Monte Carlo sample composed of 97\% nominal MC and $3 \% \mathrm{MC}$ with no accidentals produces a low-SOD fraction that is significantly different from the data. Since no significant data-MC difference in the low-SOD fraction is observed in the nominal data-MC comparison, we conclude that the effect of accidentals on tracks is modeled to within 3\%. We therefore assign a systematic uncertainty on the Dalitz branching ratio equal to $3 \%$ of $3.96 \%$, or $0.12 \%$, due to the simulation of extra tracks from accidentals.
Accidental activity results in extra "software clusters" in the CsI calorimeter. These extra clusters are found in the reconstruction but are not energetic enough to be detected by the trigger during data acquisition. The distribution of software clusters is reasonably well-modeled by the Monte Carlo simulation; the fraction of events with no extra software clusters differs by 3.3 sigma between data and Monte Carlo. A Monte Carlo sample that is composed of 99\% nominal MC and 1\% MC with no accidentals produces a data-MC mismatch in the fraction of extra software clusters that is larger than the observed data-MC discrepancy. We therefore conclude that the effect of accidentals on photon clusters in the signal mode is modeled to better than $1 \%$, and assign a systematic uncertainty on the Dalitz branching fraction equal to $1 \%$ of $3.96 \%$, or $0.04 \%$, due to the simulation of extra calorimeter clusters from accidentals.

The total uncertainty in the Dalitz branching ratio associated with the simulation of extra tracks and clusters from accidental activity is found by combining the uncertainties from extra tracks and extra clusters in quadrature. The resulting systematic uncertainty in the branching ratio is $0.13 \%$.

\section{Form factor}

The amplitude for the $\pi^{0} \rightarrow e^{+} e^{-} \gamma$ decay contains a form factor at the $\pi^{0} \gamma \gamma$ vertex. The form factor is approximated by $f(x) \approx(1+a x)$, where $x=\left(m_{e^{+} e^{-}} / m_{\pi}\right)^{2}$ and $a$ is the $\pi^{0}$ slope parameter. The nominal value of the slope parameter is $a=0.032 \pm 0.004[9,16]$. To determine the sensitivity of the Dalitz branching ratio to the value of the form factor used in the Monte Carlo simulation, we measure the change in acceptance, relative to the nominal MC, for MC samples with values of $a$ that are 8 sigma above and below the nominal value. The acceptance changes by $(0.388 \pm 0.274) \%$ and $(0.155 \pm 0.274) \%$, respectively. Dividing the larger of these two acceptance changes by 8 results in a one-sigma systematic uncertainty in the Dalitz branching ratio of $0.06 \%$.

\section{Selection criteria}

We study the systematic uncertainty associated with the analysis selection criteria by varying each selection requirement and finding the associated change in the branching ratio measurement. We find that variations in selection criteria that are common to the signal and acceptance modes cancel in the branching fraction as expected. Selection criteria that are unique to the signal mode also produce no significant change in the branching fraction when varied. Since we find no significant change in the Dalitz branching fraction when varying any of the selection criteria, we assign no additional systematic uncertainty.

We require the reconstructed $e^{+} e^{-}$mass to be greater than $15 \mathrm{MeV} / c^{2}$. Therefore, any disagreement between data and Monte Carlo in the $e^{+} e^{-}$mass scale will produce an 
uncertainty in the branching ratio measurement. We estimate our sensitivity to the $e^{+} e^{-}$mass scale by varying the scale and checking the data-MC comparison. We find that a $0.5 \%$ shift in the $e^{+} e^{-}$mass scale results in a detectable data-MC disagreement. Since we see no significant data-MC disagreement in the nominal analysis, the $e^{+} e^{-}$mass scale must match to within $0.5 \%$; the corresponding uncertainty in the branching ratio measurement is $0.06 \%$.

We require CsI calorimeter clusters to have a transverse energy distribution similar to one that is expected for a photon by applying a cut on the $\chi_{\gamma}^{2}$ variable. Since the normalization mode contains one more photon than the signal mode, any photon inefficiency resulting from this requirement is a source of systematic uncertainty. We make use of the result from a previous analysis measuring $\mathrm{B}\left(K_{L} \rightarrow \pi^{0} \pi^{0} \pi^{0}\right) / \mathrm{B}\left(K_{L} \rightarrow \pi^{ \pm} e^{\mp} \nu_{e}\right)$ [12], which has six more photons in the signal mode than in the normalization mode and found a change of $0.05 \%$ when removing the $\chi_{\gamma}^{2}$ requirement. We scale this result by $1 / 6$ to estimate the systematic uncertainty associated with the one-photon difference between the signal and normalization modes in this analysis; we assign a systematic uncertainty associated with photon inefficiency of $0.01 \%$.

\section{Background}

The primary background to the $K_{L} \rightarrow 3 \pi_{D}^{0}$ decay is $K_{L} \rightarrow \pi^{0} \pi^{0} \pi^{0}$ decay in which one photon converts to an $e^{+} e^{-}$pair at the vacuum window. Using a large sample of simulated $K_{L} \rightarrow \pi^{0} \pi^{0} \pi^{0}$ decays, we find that $0.0003 \%$ of these events are accepted by the $K_{L} \rightarrow 3 \pi_{D}^{0}$ analysis. This background is negligible, so we do not subtract it; we take the $0.0003 \%$ background rate as a systematic uncertainty on the branching ratio measurement.

TABLE II. Summary of systematic uncertainties in the $B\left(\pi^{0} \rightarrow\right.$ $\left.e^{+} e^{-} \gamma\right) / B\left(\pi^{0} \rightarrow \gamma \gamma\right)$ branching ratio.

\begin{tabular}{lr}
\hline \hline Source of uncertainty & $\begin{array}{c}\text { Level of } \\
\text { uncertainty }\end{array}$ \\
\hline Tracking inefficiency & $0.68 \%$ \\
Relative trigger inefficiency & $0.14 \%$ \\
$K_{L} \rightarrow \pi^{0} \pi^{0} \pi^{0}$ trigger inefficiency & $<0.01 \%$ \\
$K_{L} \rightarrow \pi^{0} \pi^{0} \pi^{0}$ trigger prescale & $<0.01 \%$ \\
Radiative corrections & $0.29 \%$ \\
Detector material & $0.47 \%$ \\
Extra tracks and clusters & $0.13 \%$ \\
Form factor & $0.06 \%$ \\
$e^{+} e^{-}$mass scale & $0.06 \%$ \\
Photon inefficiency & $0.01 \%$ \\
Background & $<0.01 \%$ \\
Monte Carlo statistics & $0.20 \%$ \\
Total & $0.92 \%$ \\
\hline \hline
\end{tabular}

\section{Summary of systematic uncertainties}

Table II contains a summary of the systematic uncertainties for the measurement of $B\left(\pi^{0} \rightarrow e^{+} e^{-} \gamma\right) /$ $B\left(\pi^{0} \rightarrow \gamma \gamma\right)$. Most of the sources of error are related to uncertainty in the Monte Carlo simulation of the relative acceptance between the two decay modes. The largest source of uncertainty is from differences in the tracking efficiency between data and Monte Carlo. The total systematic uncertainty on the Dalitz branching ratio measurement is $0.92 \%$.

\section{RESULT AND CROSSCHECKS}

We find 63,693 $K_{L} \rightarrow 3 \pi_{D}^{0}$ decays with an acceptance of $0.12 \%$ and $3,529,065 K_{L} \rightarrow \pi^{0} \pi^{0} \pi^{0}$ decays with an acceptance of $3.79 \%$. We scale the $K_{L} \rightarrow 3 \pi_{D}^{0}$ acceptance by a factor of 3 since any of the three pions could undergo Dalitz decay. We scale the $K_{L} \rightarrow \pi^{0} \pi^{0} \pi^{0}$ acceptance by 50 to account for the prescale applied during data collection. The final result is

$$
\begin{aligned}
& \frac{B\left(\pi^{0} \rightarrow e^{+} e^{-} \gamma\right)}{B\left(\pi^{0} \rightarrow \gamma \gamma\right)}, \quad m_{e^{+} e^{-}}>15 \mathrm{MeV} / c^{2} \\
& \quad=[3.920 \pm 0.016(\text { stat }) \pm 0.036(\text { syst })] \times 10^{-3}
\end{aligned}
$$

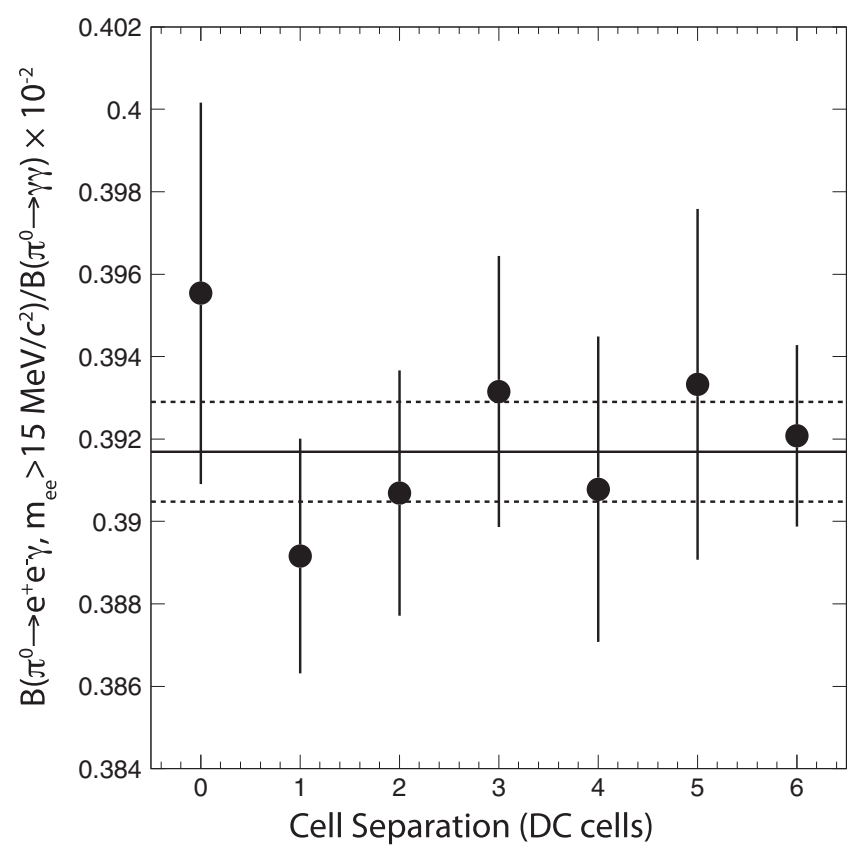

FIG. 6. The $B\left(\pi^{0} \rightarrow e^{+} e^{-} \gamma\right) / B\left(\pi^{0} \rightarrow \gamma \gamma\right)$ measurement versus cell separation. The Dalitz events that contribute to the result in each of the first six bins have a minimum cell separation equal to the bin number. The last bin includes events with minimum cell separation greater than or equal to six. The error bars represent the independent statistical uncertainty in each bin. The solid line is the weighted average, and the dashed horizontal lines indicate the statistical uncertainty on the weighted average. 
We check the consistency of the $B\left(\pi^{0} \rightarrow e^{+} e^{-} \gamma\right) /$ $B\left(\pi^{0} \rightarrow \gamma \gamma\right)$ branching ratio measurement by comparing the result among subsets of the data. We separate the data into groups by cell separation, $e^{+} e^{-}$mass, beam intensity, time, whether the tracks bend toward or away from each other, and the two polarities of the analysis magnet. In each case, the results in the subsamples agree with each other and with the result from the full data sample. Figure 6 shows the $B\left(\pi^{0} \rightarrow e^{+} e^{-} \gamma\right) / B\left(\pi^{0} \rightarrow \gamma \gamma\right) \quad\left(m_{e^{+} e^{-}}>15 \mathrm{MeV} / c^{2}\right)$ result as a function of minimum cell separation with the track separation requirement of three cells removed. A constant fit to these points has a probability of $92 \%$; the result is stable as a function of minimum cell separation.

We correct the result in Eq. (3), which is valid for $e^{+} e^{-}$ masses greater than $15 \mathrm{MeV} / c^{2}$, to the full mass range using a calculation of the $e^{+} e^{-}$mass spectrum from Mikaelian and Smith [4]. We find that $33.9128 \%$ of Dalitz decays occur above the $15 \mathrm{MeV} / c^{2} e^{+} e^{-}$mass cutoff applied in this analysis. The corrected result, valid over the full $e^{+} e^{-}$mass range, is

$$
\begin{aligned}
& \frac{B\left(\pi^{0} \rightarrow e^{+} e^{-} \gamma\right)}{B\left(\pi^{0} \rightarrow \gamma \gamma\right)} \\
& \quad=[1.1559 \pm 0.0047(\text { stat }) \pm 0.0106(\text { syst })] \% .
\end{aligned}
$$

\section{CONCLUSION}

We have measured the Dalitz decay branching ratio, $B\left(\pi^{0} \rightarrow e^{+} e^{-} \gamma\right) / B\left(\pi^{0} \rightarrow \gamma \gamma\right)$, for $e^{+} e^{-}$masses greater than $15 \mathrm{MeV} / c^{2}$ using $K_{L} \rightarrow 3 \pi_{D}^{0}$ and $K_{L} \rightarrow \pi^{0} \pi^{0} \pi^{0}$ decays. Correcting to the full $e^{+} e^{-}$mass range, we find

$$
\frac{B\left(\pi^{0} \rightarrow e^{+} e^{-} \gamma\right)}{B\left(\pi^{0} \rightarrow \gamma \gamma\right)}=(1.1559 \pm 0.0116) \%
$$

Figure 7 is a comparison of this result to the theoretical calculation and previous experimental results. This result agrees with the 1972 theoretical calculation at the 2.4 sigma level, where a $1 \%$ uncertainty on the calculation has been assumed based on discussion in [4]. The discrepancy with [8] is 3.6 sigma. The uncertainty in

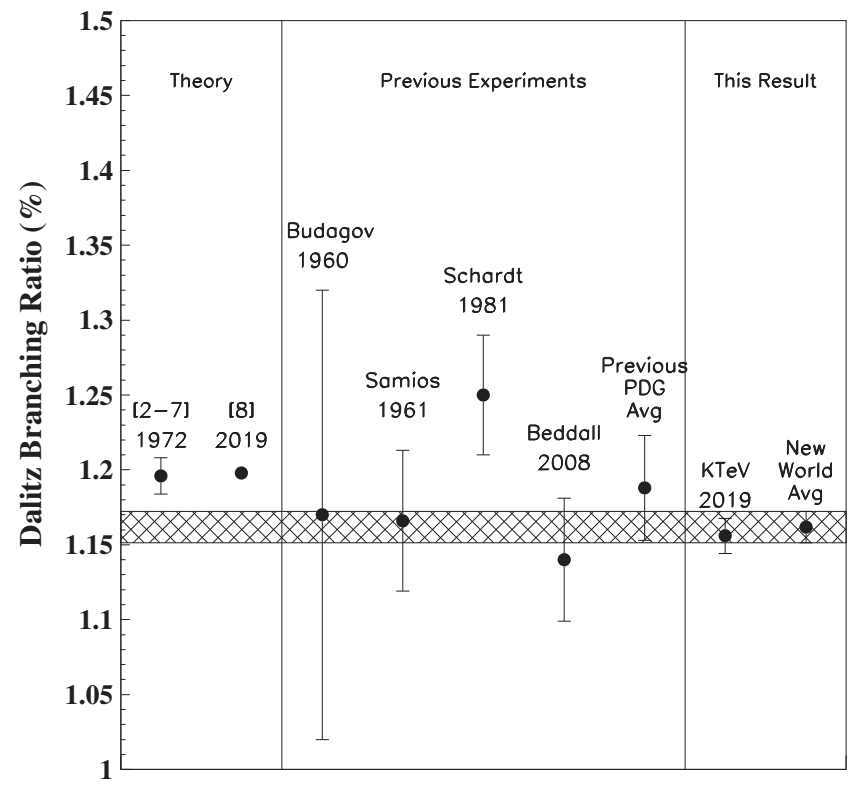

FIG. 7. Comparison of the $B\left(\pi^{0} \rightarrow e^{+} e^{-} \gamma\right) / B\left(\pi^{0} \rightarrow \gamma \gamma\right)$ branching ratio result to previous theoretical [2-8] and experimental $[9,18-21]$ results. A new world average for experimental results is also shown. The hatched region shows the area within one sigma of the new world average.

this measurement is at least a factor of 3 smaller than the individual uncertainties on all previous measurements and the uncertainty on the previous particle data group average [9]. We combine this result with the four previous measurements to find the new world average is $B\left(\pi^{0} \rightarrow e^{+} e^{-} \gamma\right) / B\left(\pi^{0} \rightarrow \gamma \gamma\right)=(1.1619 \pm 0.0105) \%$.

\section{ACKNOWLEDGMENTS}

We gratefully acknowledge the support and effort of the Fermilab staff and the technical staffs of the participating institutions for their vital contributions. This work was supported in part by the U.S. Department of Energy, The National Science Foundation, The Ministry of Education and Science of Japan, Fundação de Amparo a Pesquisa do Estado de São Paulo-FAPESP, Conselho Nacional de Desenvolvimento Cientifico e Tecnologico-CNPq, and CAPES-Ministerio Educação.
[1] R. Dalitz, Proc. Phys. Soc. London Sect. A 64, 667 (1951).

[2] D. Joseph, Nuovo Cimento 16, 997 (1960).

[3] B. Lautrup and J. Smith, Phys. Rev. D 3, 1122 (1971).

[4] K. Mikaelian and J. Smith, Phys. Rev. D 5, 1763 (1972).
[5] K. Kampf, J. Novotny, and M. Knecht, in Hadron Structure. Proceedings, International Conference, Herlany, Slovakia (2002), pp. 67-73, https://arxiv.org/abs/hep-ph/0212243.

[6] K. Kampf, M. Knecht, and J. Novotny, Eur. Phys. J. C 46, 191 (2006). 
[7] T. Husek, K. Kampf, and J. Novotny, Phys. Rev. D 92, 054027 (2015).

[8] T. Husek, E. Goudzovski, and K. Kampf, Phys. Rev. Lett. 122, 022003 (2019).

[9] C. Patrignani et al. (Particle Data Group), Chin. Phys. C 40, 100001 (2016).

[10] E. Abouzaid et al. (KTeV Collaboration), Phys. Rev. D 83, 092001 (2011).

[11] A. Alavi-Harati et al. (KTeV Collaboration), Phys. Rev. D 67, 012005 (2003); 70, 079904(E) (2004).

[12] T. Alexopoulos et al. (KTeV Collaboration), Phys. Rev. D 70, 092006 (2004).

[13] A. J. Malensek, Fermilab Report No. FN-341 (Errata), 1981, http://inspirehep.net/record/169381.

[14] R. Brun et al., GEANT 3.21 (CERN, Geneva, 1994), http:// inspirehep.net/record/252007/.
[15] The $\chi_{\gamma}^{2}$ variable is not normalized to be a true chi-squared.

[16] Since the analysis described here was performed, a new measurement of the slope parameter has been made by NA62 [17]. The change to the world average of the form factor is +0.003 with respect to the value used in this analysis; that difference is covered by the systematic uncertainty quoted here.

[17] C. Lazzeroni et al. (NA62 Collaboration), Phys. Lett. B 768, 38 (2017).

[18] Y. A. Budagov et al., JETP Lett. 11, 755 (1960).

[19] N. Samios, Phys. Rev. 121, 275 (1961).

[20] M. Schardt, J. S. Frank, C. M. Hoffman, R. E. Mischke, D. C. Moir, and P. A. Thompson, Phys. Rev. D 23, 639 (1981).

[21] A. Beddall, Eur. Phys. J. C 54, 365 (2008). 